\title{
Control of Generalized Contact Motion and Force in Physical Human-Robot Interaction
}

\author{
Emanuele Magrini Fabrizio Flacco Alessandro De Luca
}

\begin{abstract}
During human-robot interaction tasks, a human may physically touch a robot and engage in a collaboration phase with exchange of contact forces and/or requiring coordinated motion of a common contact point. Under the premise of keeping the interaction safe, the robot controller should impose a desired motion/force behavior at the contact or explicitly regulate the contact forces. Since intentional contacts may occur anywhere along the robot structure, the ability of controlling generalized contact motion and force becomes an essential robot feature. In our recent work, we have shown how to estimate contact forces without an explicit force sensing device, relying on residual signals to detect contact and on the use of an external (depth) sensor to localize the contact point. Based on this result, we introduce two control schemes that generalize the impedance and direct force control paradigms to a generic contact location on the robot, making use of the estimated contact forces. The issue of human-robot task compatibility is pointed out in case of control of generalized contact forces. Experimental results are presented for a KUKA LWR robot using a Kinect sensor.
\end{abstract}

\section{INTRODUCTION}

In the robotics community at large, there is great excitement about the recent possibility of realizing safe physical collaboration between human users and a new industrial generation of lightweight, compliant, and friendly robots [1], [2]. This technical achievement is the outcome of advances in mechanics, actuation, sensing, and control that have emerged in the last few years, from statements of basic requirements (see, e.g., [3]-[6]) to novel research results (see, e.g., [7], [8] for the original collision detection algorithm implemented in the KUKA LWR and iiwa robots). Indeed, more of such scientific developments and technological transfers are needed in order to improve further the autonomy, safety, and ease of use of robotic coworkers in industrial settings and of personal assistants in service applications.

Along this path, we have proposed a control architecture for safer physical human-robot collaboration, based on a hierarchy of three consistent robot behaviors [9]. In this framework, safety is the innermost layer with the highest priority of execution: it implies the ability of detecting and fast reacting to accidental collisions [10] and distinguishing between these and intentional contacts [11], [12]; coexistence is the robot capability of sharing workspaces, without interfering with humans: it calls for collision avoidance features [13], [14], and robot operative flexibility for

The authors are with the Dipartimento di Ingegneria Informatica, Automatica e Gestionale, Sapienza Università di Roma, Via Ariosto 25, 00185 Roma, Italy (\{magrini,fflacco,deluca $\} @$ diag.uniroma1.it). This work is supported by the European Commission, within the FP7 ICT-287513 SAPHARI project (www.saphari.eu). modifying on line, abandoning, and resuming the originally planned tasks; finally, collaboration requires the robot to perform complex tasks involving direct physical interaction and coordination with humans.

One can consider different possible collaborative tasks where physical human-robot interaction occurs: carrying a heavy payload, handing over objects, manipulating limp materials, holding firmly a part while the partner is operating on it, and so on. In most cases, regulation of the forces exchanged at the contact is needed and/or accurate control of relative motion while keeping in place the original contact. As additional convenient features, whole-body manipulation should be possible, with single or multiple contacts occurring anywhere along the robot structure, and a minimum amount of extra sensing should be requested, in particular without the need of a F/T sensor at the wrist or of distributed tactile sensing for the measurement of contact/interaction forces.

In [15], a method has been developed for estimating the interaction forces (in direction and intensity) during dynamic contacts between a robot and a human, with no explicit force/torque sensing. The method is based on the integrated use of model-based residual signals that detect the occurrence of collisions [8] and of one or more external RGB-D sensors (e.g., Kinects) to approximately localize the contact point on the surface of the robot links. This 'virtual force sensor' was shown to perform with reasonably good estimation accuracy. Its output has been used already in a series of humanrobot interaction experiments based on an admittance control scheme defined at the contact point.

In this paper, we illustrate further the use of the estimated contact forces for the design of two human-robot interaction controllers that extend the impedance control method [16] and the direct force control method [17], respectively to the case of generic contact locations on the robot. For this reason, we collectively denote this approach as generalized contact motion and force control.

While direct force control needs always a measure (or an estimate) of the contact force, standard joint or Cartesian impedance control laws require a force measure only if the natural robot inertia (at the proper level) has to be changed to a desired one. When the robot inertia is left unchanged, force sensing is no longer required (the term compliance control should be used then). The same situation holds true also for our generalized contact impedance control laws, simply replacing actual measurements with the use of force estimates (see also Fig. 1).

When realizing a force control law at the contact level, task compatibility issues may arise, mainly due to the 




Fig. 1. A schematic comparison of impedance control schemes for a fully rigid robot arm, when a desired mass-spring-damper behavior is imposed at the joint-level, at the Cartesian-level, or at the proposed contact-level, in response to a contact force $\boldsymbol{F}_{c}$. The estimated contact force $\widehat{\boldsymbol{F}}_{c}$ can be obtained with the method in [15]).

generality of the possible locations of the contact point. A first reason follows from the arguments already presented in [15]. When contacts occur on a link close to the robot base, the estimation method may have too little information to recover a complete estimate of the applied contact force (in particular, the contact Jacobian may not be full rank). The second reason is even more fundamental, and refers to the type of engaged human-robot contact and its compatibility with the specification of a desired contact force. A complete specification of three independent scalar references for the contact force may be incompatible with the way the human is pushing on the robot. The situation is similar to defining a correct task frame in hybrid force/motion control [18], [19, Chap. 9.6-7]: the robot cannot control forces along contact directions where the human is not providing a reaction.

While the contact admittance control scheme in [15] specifies joint velocity references in response to estimated contact forces, and relies upon low-level servo loops for their execution, the two control designs presented in this paper generate torque commands, and assume thus a torquecontrolled robot (viz., motor currents can be imposed). In addition, an accurate robot dynamic model will be needed for control implementation. All developments in the paper are presented for rigid robot manipulators. Accordingly, no joint torque sensors are used (nor needed) and the 'virtual force sensor' concept and the associated contact force/impedance controllers can be implemented using only the joint encoders as proprioceptive sensing.

On the other hand, the presented control experiments have been performed on a KUKA LWR where the use of harmonic drive introduces joint elasticity [20]. In practice, however, the control implementation for this robot is fully transparent with respect to the presence of compliant joints. The user defined torque-controlled modality of the FastResearchInterface (FRI) [21] implements the torque controller originally proposed in [22], and allows to use the available joint torque sensors both for residual computation and for control. Moreover, for all model-based computations we have used our own identified dynamic model of the LWR link dynamics [23].

The paper is organized as follows. Section II sets the notation and includes a short summary on the contact force estimation method [15] used in the remainder of the paper. Section III recaps in a compact way the standard impedance and direct force control laws for a single mass subject to an external contact force. The generalization of these control schemes to handle contact situations occurring at a generic point of a multi-dof robot manipulator is detailed in Sec. IV. Section V reports on several experimental results obtained with the proposed control methods during physical humanrobot interaction with a KUKA LWR 4 robot and a Kinect sensor.

\section{PRELiminaries}

For a robot manipulator having $n$ serial joints, with associated generalized coordinates $\boldsymbol{q} \in \mathbb{R}^{n}$, assume that a motion/force interaction task is defined for a contact point $\boldsymbol{x}_{c} \in \mathbb{R}^{3}$ whose direct kinematics map is given by $\boldsymbol{x}_{c}=$ $\boldsymbol{f}(\boldsymbol{q})$. Differentiating this twice w.r.t. time yields

$$
\ddot{\boldsymbol{x}}_{c}=\boldsymbol{J}_{c}(\boldsymbol{q}) \ddot{\boldsymbol{q}}+\dot{\boldsymbol{J}}_{c}(\boldsymbol{q}) \dot{\boldsymbol{q}}
$$

where $\boldsymbol{J}_{c}=\partial \boldsymbol{f}(\boldsymbol{q}) / \partial \boldsymbol{q}$ is the $3 \times n$ contact Jacobian matrix. Note that if the contact point is located on link $k$ ( $k=$ $1, \ldots, n)$, the last $n-k$ columns of $\boldsymbol{J}_{c}$ will be identically zero. In most cases, the robot is redundant respect to the given interaction task, being $k>3$.

At a given robot state $(\boldsymbol{q}, \dot{\boldsymbol{q}})$, all joint accelerations associated to a desired acceleration $\ddot{\boldsymbol{x}}_{c}$ of the contact point can be written as

$$
\ddot{\boldsymbol{q}}=\boldsymbol{J}_{c}^{\#}\left(\ddot{\boldsymbol{x}}_{c}-\dot{\boldsymbol{J}}_{c} \dot{\boldsymbol{q}}\right)+\boldsymbol{P}_{c} \ddot{\boldsymbol{q}}_{0},
$$

where $\boldsymbol{J}_{c}^{\#}$ is a $n \times 3$ generalized inverse of the contact Jacobian, $\ddot{\boldsymbol{q}}_{0} \in \mathbb{R}^{n}$ is an arbitrary joint acceleration, and $\boldsymbol{P}_{c}=\boldsymbol{I}-\boldsymbol{J}_{c}^{\#} \boldsymbol{J}_{c}$ is a projector in the null space of $\boldsymbol{J}_{c}$.

The dynamic model of a rigid manipulator interacting with the (human) environment at a robot point $\boldsymbol{x}_{c}$ is given by

$$
\boldsymbol{M}(\boldsymbol{q}) \ddot{\boldsymbol{q}}+\boldsymbol{C}(\boldsymbol{q}, \dot{\boldsymbol{q}}) \dot{\boldsymbol{q}}+\boldsymbol{g}(\boldsymbol{q})=\boldsymbol{\tau}+\boldsymbol{J}_{c}^{T}(\boldsymbol{q}) \boldsymbol{F}_{c},
$$

with positive definite inertia matrix $\boldsymbol{M}$, Coriolis and centrifugal velocity terms $\boldsymbol{C} \dot{\boldsymbol{q}}$ (factorized by the Christtoffel symbols), gravitational terms $\boldsymbol{g}$, control torque $\boldsymbol{\tau} \in \mathbb{R}^{n}$, and joint torque $\boldsymbol{\tau}_{c}=\boldsymbol{J}_{c}^{T}(\boldsymbol{q}) \boldsymbol{F}_{c}$ resulting from the contact interaction force $\boldsymbol{F}_{c} \in \mathbb{R}^{3}$. We will use also the compact notation $\boldsymbol{n}(\boldsymbol{q} \cdot \dot{\boldsymbol{q}})=\boldsymbol{C}(\boldsymbol{q}, \dot{\boldsymbol{q}}) \dot{\boldsymbol{q}}+\boldsymbol{g}(\boldsymbol{q})$.

In the framework of operational space control [18], it has been shown that using the inertia-weighted pseudoinverse as the generalized inverse in (2) guarantees consistency of 
the transformation of task forces in the robot dynamics. The inertia-weighted pseudoinverse of contact Jacobian is

$$
\boldsymbol{J}_{c, M}^{\#}=\boldsymbol{M}^{-1} \boldsymbol{J}_{c}^{T}\left(\boldsymbol{J}_{c} \boldsymbol{M}^{-1} \boldsymbol{J}_{c}^{T}\right)^{-1} .
$$

In the following, to simplify notation, each pseudoinverse $\boldsymbol{J}_{c}^{\#}$ is to be intended as the inertia-weighted one given by (4).

\section{A. Contact force estimation}

The residual vector $\boldsymbol{r} \in \mathbb{R}^{n}$ for a robot with dynamics (3) is defined as [7]

$$
\boldsymbol{r}(t)=\boldsymbol{K}_{I}\left(\boldsymbol{p}-\int_{0}^{t}\left(\boldsymbol{\tau}+\boldsymbol{C}^{T}(\boldsymbol{q}, \dot{\boldsymbol{q}}) \dot{\boldsymbol{q}}-\boldsymbol{g}(\boldsymbol{q})+\boldsymbol{r}\right) d s\right)
$$

where $\boldsymbol{p}=\boldsymbol{M}(\boldsymbol{q}) \dot{\boldsymbol{q}}$ is the generalized momentum of the robot and $\boldsymbol{K}_{I}>0$ is a diagonal gain matrix. The dynamic evolution of $\boldsymbol{r}$ has the stable, first-order filter structure

$$
\dot{\boldsymbol{r}}=\boldsymbol{K}_{I}\left(\boldsymbol{\tau}_{c}-\boldsymbol{r}\right)
$$

Thus, for sufficiently large gains, we can assume that

$$
\boldsymbol{r} \simeq \boldsymbol{\tau}_{c}=\boldsymbol{J}_{c}^{T}(\boldsymbol{q}) \boldsymbol{F}_{c}
$$

Equation (6) forms the basis for the estimations of the unknown contact force $\boldsymbol{F}_{c} \in \mathbb{R}^{3}$. Using external sensing, the location of the contact point $\boldsymbol{x}_{c}$ can be determined, and thus the associated contact Jacobian $\boldsymbol{J}_{c}$ can be computed. Depending on which link in the kinematic chain is involved in the contact, (6) may consist of a square, under-, or overdetermined linear system. In any case, the contact force is estimated by pseudoinversion as

$$
\widehat{\boldsymbol{F}}_{c}=\left(\boldsymbol{J}_{c}^{T}(\boldsymbol{q})\right)^{\#} \boldsymbol{r}
$$

Indeed, the estimate $\widehat{\boldsymbol{F}}_{c}$ will be limited only to those components of $\boldsymbol{F}_{c}$ that can be detected by the residual $\boldsymbol{r}$, namely those contact forces that do not belong to the null space $\mathcal{N}\left(\boldsymbol{J}_{c}^{T}(\boldsymbol{q})\right)$. For further details and for the analysis of cases when the contact Jacobian is not full rank, see [15].

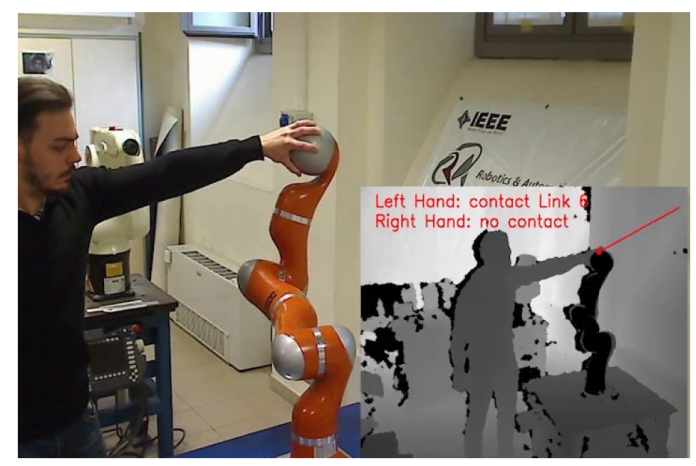

Fig. 2. A view of the result of the on-line estimation of the contact force

\section{IMPEDANCE AND FORCE CONTROL FOR A SINGLE MASS}

As an introduction to the control design for the general multi-dof robotic case, consider first a very simple 1-dof example. As shown in Fig. 3, a single mass $m$ moves on a frictionless horizontal plane under the action of a control force $f$ and of a contact force $f_{c}$. The dynamic equation is

$$
m \ddot{x}=f+f_{c} .
$$

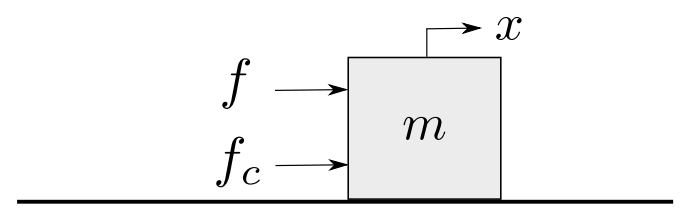

Fig. 3. A mass $m$ subject to a control force $f$ and a contact force $f_{c}$.

\section{A. Impedance control}

The ‘inverse dynamics' control law

$$
f=m a-f_{c},
$$

transforms system (8) into the double integrator

$$
\ddot{x}=a \text {. }
$$

The auxiliary input $a$ has to be designed so that the controlled mass $m$, under the action of the contact force $f_{c}$, matches the behavior of an impedance model characterized by a desired (apparent) mass $m_{d}>0$, desired damping $k_{d}>0$, and desired stiffness $k_{p}>0$, all with respect to a smooth motion reference $x_{d}(t)$, or

$$
m_{d}\left(\ddot{x}-\ddot{x}_{d}\right)+k_{d}\left(\dot{x}-\dot{x}_{d}\right)+k_{p}\left(x-x_{d}\right)=f_{c} .
$$

This is obtained by using the control force

$f=\frac{m}{m_{d}}\left(\ddot{x}_{d}+k_{d}\left(\dot{x}_{d}-\dot{x}\right)+k_{p}\left(x_{d}-x\right)\right)+\left(\frac{m}{m_{d}}-1\right) f_{c}$.

For $k_{d}^{2}<4 k_{p} m_{d}$, the second-order linear system (11) is characterized by a pair of asymptotically stable complex poles with natural frequency and damping ratio given by

$$
\omega_{n}=\sqrt{\frac{k_{p}}{m_{d}}}, \quad \zeta=\frac{k_{d}}{2 \sqrt{k_{p} m_{d}}} .
$$

Reducing the desired mass $m_{d}$, for given values of stiffness and damping, will increase both the angular frequency and the damping ratio, and thus improve transients. On the other hand, for a given mass, an increase of the stiffness $k_{p}$ should be accompanied by an increase of the damping $k_{d}$ in order to prevent more oscillatory transients.

If the desired mass equals the natural (original) mass, i.e., $m_{d}=m$, a measure of the contact force $f_{c}$ is no longer needed in the impedance controller (12) —which is then also called a compliance control law, since the main design parameter left is the desired stiffness $k_{p}$. In particular, for 
regulation tasks (with $x_{d}(t)=x_{d}=$ constant) the control law collapses just to a PD action on the position error $e=x_{d}-x$,

$$
f=k_{p}\left(x_{d}-x\right)-k_{d} \dot{x} .
$$

The system will converge to $x=x_{d}$ if and only if there is no contact force. With $f_{c} \neq 0$ but constant, there will be convergence to the closed-loop equilibrium $x_{e}$ satisfying

$$
k_{p}\left(x_{d}-x_{e}\right)+f_{c}=0 \quad \Rightarrow \quad x_{e}=x_{d}+\frac{f_{c}}{k_{p}} .
$$

\section{B. Force control}

If we desire to control directly the contact force to a desired (possibly, constant) value $f_{d}$, it is necessary to build a force error $e_{f}=f_{d}-f_{c}$ into the control law. After using eq. (9), define the auxiliary input $a$ as

$$
a=\frac{1}{m_{d}}\left(k_{f}\left(f_{d}-f_{c}\right)-k_{d} \dot{x}\right)
$$

with force error gain $k_{f}>0$ and velocity damping $k_{d}>0$. The associated control force is then

$$
f=\frac{m}{m_{d}}\left(k_{f}\left(f_{d}-f_{c}\right)-k_{d} \dot{x}\right)-f_{c} .
$$

A contact force measure is always needed in this case, even if we choose $m_{d}=m$. The closed-loop system becomes

$$
m_{d} \ddot{x}+k_{d} \dot{x}=k_{f}\left(f_{d}-f_{c}\right),
$$

which shows that the force error $e_{f}$ goes to zero if a constant contact force is applied. However, the actual position $x=x_{e}$ reached at the equilibrium is not known a priori, and will depend on the actual history of the external contact force.

\section{Generalized Contact Motion and Force CONTROL FOR ROBOTS}

We extend here the control design results of Sec. III to a generic robot contact point $\boldsymbol{x}_{c}$ of a robot manipulator, and consider also the use of the contact force estimation $\widehat{\boldsymbol{F}}_{c}$, as provided by (7), in place of the real measurement, each time this will be needed.

\section{A. Impedance control}

Starting from robot model (3), and dropping from now on dependencies for the sake of compactness, consider the inverse dynamics control law

$$
\boldsymbol{\tau}=\boldsymbol{M a}+\boldsymbol{n}-\boldsymbol{J}_{c}^{T} \widehat{\boldsymbol{F}}_{c},
$$

where $\widehat{\boldsymbol{F}}_{c}$ is the estimated contact force. In ideal design conditions, the above control law realizes a feedback linearization, leading to a system of double integrators $\ddot{\boldsymbol{q}}=\boldsymbol{a}$. The auxiliary control input $\boldsymbol{a}$ is chosen so as to impose a mechanical impedance model between the (estimated) contact forces $\widehat{\boldsymbol{F}}_{c}$ and the motion of the contact point $\boldsymbol{x}_{c}$, or

$$
\boldsymbol{M}_{d}\left(\ddot{\boldsymbol{x}}_{c}-\ddot{\boldsymbol{x}}_{d}\right)+\boldsymbol{K}_{d}\left(\dot{\boldsymbol{x}}_{c}-\dot{\boldsymbol{x}}_{d}\right)+\boldsymbol{K}_{p}\left(\boldsymbol{x}_{c}-\boldsymbol{x}_{d}\right)=\widehat{\boldsymbol{F}}_{c}
$$

being $\boldsymbol{M}_{d}>0$ the desired inertia matrix, $\boldsymbol{K}_{d}>0$ the desired damping matrix, $\boldsymbol{K}_{p}>0$ the desired stiffness matrix (very often, these matrices are taken as diagonal), and $\boldsymbol{x}_{d}(t)$ the smooth desired trajectory of the contact point. Solving for $\ddot{\boldsymbol{x}}_{c}$ from (20) and using (2), the control input $\boldsymbol{a}$ is obtained as

$\boldsymbol{a}=\boldsymbol{J}_{c}^{\#} \boldsymbol{M}_{d}^{-1}\left(\boldsymbol{M}_{d} \ddot{\boldsymbol{x}}_{d}+\boldsymbol{K}_{d} \dot{\boldsymbol{e}}+\boldsymbol{K}_{p} \boldsymbol{e}-\boldsymbol{M}_{d} \dot{\boldsymbol{J}}_{c} \dot{\boldsymbol{q}}+\widehat{\boldsymbol{F}}_{c}\right)+\boldsymbol{P} \ddot{\boldsymbol{q}}_{0}$,

with contact position error $\boldsymbol{e}=\boldsymbol{x}_{d}-\boldsymbol{x}_{c}$. Thus, the resulting torque control input is

$$
\begin{aligned}
\boldsymbol{\tau}= & \boldsymbol{M} \boldsymbol{J}_{c}^{\#} \boldsymbol{M}_{d}^{-1}\left(\boldsymbol{M}_{d} \ddot{\boldsymbol{x}}_{d}+\boldsymbol{K}_{d} \dot{\boldsymbol{e}}+\boldsymbol{K}_{p} \boldsymbol{e}-\boldsymbol{M}_{d} \dot{\boldsymbol{J}}_{c} \dot{\boldsymbol{q}}+\widehat{\boldsymbol{F}}_{c}\right) \\
& +\boldsymbol{M} \boldsymbol{P} \ddot{\boldsymbol{q}}_{0}+\boldsymbol{n}-\boldsymbol{J}_{c}^{T} \widehat{\boldsymbol{F}}_{c} .
\end{aligned}
$$

In (22), observe that the contribution of $\widehat{\boldsymbol{F}}_{c}$ is given by

$$
\boldsymbol{\tau}=\ldots+\left(\boldsymbol{M} \boldsymbol{J}_{c}^{\#} \boldsymbol{M}_{d}^{-1}-\boldsymbol{J}_{c}^{T}\right) \widehat{\boldsymbol{F}}_{c} .
$$

As expected, when choosing the desired inertia matrix $\boldsymbol{M}_{d}$ equal to the natural Cartesian inertia of the robot at the contact point, also the estimation of the contact forces $\widehat{\boldsymbol{F}}_{c}$ will no longer be needed. At a given configuration $\boldsymbol{q}$, the natural inertia of the robot at a generic contact point is

$$
M_{d}=\left(\boldsymbol{J}_{c} \boldsymbol{M}^{-1} \boldsymbol{J}_{c}^{T}\right)^{-1} .
$$

By using (4), it is easy to see that $\boldsymbol{M} \boldsymbol{J}_{c}^{\#} \boldsymbol{M}_{d}^{-1}-\boldsymbol{J}_{c}^{T}=\mathbf{0}$. Whenever we desire to impose a different inertial behavior at the contact, e.g., so as to achieve different behaviors in different directions of the operative space, we shall make use of the contact force estimate $\widehat{\boldsymbol{F}}_{c}$.

\section{B. Force control}

The impedance method realizes only an indirect control of the forces exchanged during the interaction with the human, dynamic balancing with the desired position $\boldsymbol{x}_{c}$ of the contact point. The development of a direct controller for the contact force is crucial for collaborative tasks that needs accurate execution in uncertain conditions.

With the reference to (19), choose in place of the auxiliary command (21), the following acceleration

$\boldsymbol{a}=\boldsymbol{J}_{c}^{\#} \boldsymbol{M}_{d}^{-1}\left(\boldsymbol{K}_{f}\left(\boldsymbol{F}_{d}-\widehat{\boldsymbol{F}}_{c}\right)-\boldsymbol{K}_{d} \dot{\boldsymbol{x}}_{c}-\boldsymbol{M}_{d} \dot{\boldsymbol{J}}_{c} \dot{\boldsymbol{q}}\right)+\boldsymbol{P} \ddot{\boldsymbol{q}}_{0}$,

where $\boldsymbol{F}_{d}$ is the desired force at the contact point, the contact force error is $\boldsymbol{e}_{f}=\boldsymbol{F}_{d}-\widehat{\boldsymbol{F}}_{c}$, and $\boldsymbol{K}_{f}>0$ is the force error gain matrix. Replacing (25) in (19), the final torque control input is

$$
\begin{aligned}
\boldsymbol{\tau}= & \boldsymbol{M} \boldsymbol{J}_{c}^{\#} \boldsymbol{M}_{d}^{-1}\left(\boldsymbol{K}_{f} \boldsymbol{e}_{f}-\boldsymbol{K}_{d} \dot{\boldsymbol{x}}_{c}-\boldsymbol{M}_{d} \dot{\boldsymbol{J}}_{c} \dot{\boldsymbol{q}}\right) \\
& +\boldsymbol{M} \boldsymbol{P} \ddot{\boldsymbol{q}}_{0}+\boldsymbol{n}-\boldsymbol{J}_{c}^{T} \widehat{\boldsymbol{F}}_{c} .
\end{aligned}
$$

Similarly to (18), the closed-loop system is described by

$$
\boldsymbol{M}_{d} \ddot{\boldsymbol{x}}_{c}+\boldsymbol{K}_{d} \dot{\boldsymbol{x}}_{c}=\boldsymbol{K}_{f}\left(\boldsymbol{F}_{d}-\widehat{\boldsymbol{F}}_{c}\right),
$$

which shows again that the force error should go to zero whenever a constant contact force is applied. Nonetheless, the issue of task compatibility remains open. In the above expression of the control law for the human-robot contact forces, the specification of the desired force $\boldsymbol{F}_{d}$ is apparently an unconstrained one. We shall see in the experiments that 
a careful choice of $\boldsymbol{F}_{d}$ should be made so as to avoid inconsistent and drifting behavior of the robot, with serious consequences for safety.

\section{EXPERIMENTAL RESULTS}

Dynamic human-robot interaction experiments using the proposed controllers have been performed on a KUKA LWR 4. The workspace is monitored by a Microsoft Kinect depth sensor, positioned at a distance of $1.7 \mathrm{~m}$ behind the robot and at a height of $1.1 \mathrm{~m}$ w.r.t. the robot base frame. The Kinect provides $640 \times 480$ depth images at $30 \mathrm{~Hz}$ rate. All algorithms are executed on a quad-core CPU. The complete process of contact force estimation and motion and/or force control run on the robot at $5 \mathrm{~ms}$ cycle time.

With reference to the impedance control law (22), define $\boldsymbol{x}_{d}=\boldsymbol{x}_{c}\left(t_{c}\right) \in \mathbb{R}^{3}$ as the initial (and constant) position of the contact point when the interaction with the human begins at $t=t_{c}$. The desired velocity and acceleration are set then to zero, $\dot{x}_{d}=\ddot{\boldsymbol{x}}_{d}=\mathbf{0}$. Due to the redundancy of this robot with respect to many tasks, a null-space acceleration vector has been chosen as $\ddot{\boldsymbol{q}}_{0}=-\boldsymbol{K}_{N} \dot{\boldsymbol{q}}$, with $\boldsymbol{K}_{N}>0$, in order to bound/damp out self-motion movement of the arm. Finally, recall that the KUKA LWR 4 has a built-in gravity compensation. Thus, the actual impedance control law used to command the robot was

$$
\begin{aligned}
\boldsymbol{\tau}= & \boldsymbol{M} \boldsymbol{J}_{c}^{\#} \boldsymbol{M}_{d}^{-1}\left(\boldsymbol{K}_{p} \boldsymbol{e}-\boldsymbol{K}_{d} \dot{\boldsymbol{x}}_{c}-\boldsymbol{M}_{d} \dot{\boldsymbol{J}}_{c} \dot{\boldsymbol{q}}+\widehat{\boldsymbol{F}}_{c}\right) \\
& -\boldsymbol{M P} \boldsymbol{K}_{N} \dot{\boldsymbol{q}}+\boldsymbol{C} \dot{\boldsymbol{q}}-\boldsymbol{J}_{c}^{T} \widehat{\boldsymbol{F}}_{c} .
\end{aligned}
$$

Similarly for the force control law it is

$$
\begin{aligned}
\boldsymbol{\tau}= & \boldsymbol{M} \boldsymbol{J}_{c}^{\#} \boldsymbol{M}_{d}^{-1}\left(\boldsymbol{K}_{f} \boldsymbol{e}_{f}-\boldsymbol{K}_{d} \dot{\boldsymbol{x}}_{c}-\boldsymbol{M}_{d} \dot{\boldsymbol{J}}_{c} \dot{\boldsymbol{q}}\right) \\
& -\boldsymbol{M} \boldsymbol{P} \boldsymbol{K}_{N} \dot{\boldsymbol{q}}+\boldsymbol{C} \dot{\boldsymbol{q}}-\boldsymbol{J}_{c}^{T} \widehat{\boldsymbol{F}}_{c} .
\end{aligned}
$$

All experiments presented in this paper are recorded in the accompanying video clip. The plots can be even better appreciated when looking in parallel at the video.

\section{A. Impedance control with natural contact inertia}

In the first experiment, the human pushes the robot at different contact points and on different links, as shown in the snapshot of Fig. 4. The control law is given by (28), with the desired inertia matrix at the contact chosen equal to (24). The other impedance parameters were set to $\boldsymbol{K}_{d}=80 \cdot \boldsymbol{I}_{3}$, $\boldsymbol{K}_{p}=500 \cdot \boldsymbol{I}_{3}$, and $\boldsymbol{K}_{N}=20 \cdot \boldsymbol{I}_{7}$, where $\boldsymbol{I}_{k}$ denotes the $k \times k$ identity matrix. Figure 5 shows the behavior of the residual vector, of the contact force estimate, and of the Cartesian position error of the contact point with respect to its initial position $\boldsymbol{x}_{d}=\boldsymbol{x}_{c}\left(t_{c}\right)$.

\section{B. Impedance control with modified contact inertia}

In this experiment, the impedance scheme (28) was used again during the human-robot interaction. However, the desired inertia matrix at the contact point was set to

$$
\boldsymbol{M}_{d}=\left(\begin{array}{ccc}
20 & 0 & 0 \\
0 & 3 & 0 \\
0 & 0 & 10
\end{array}\right),
$$

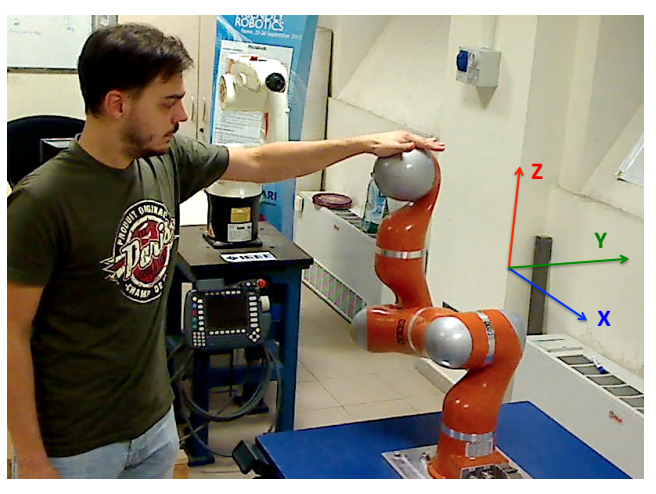

Fig. 4. The set-up and the Cartesian reference frame for the contact impedance control experiments. The color of the $X, Y$, and $Z$ axes are the same used for the associated components in the following plots.
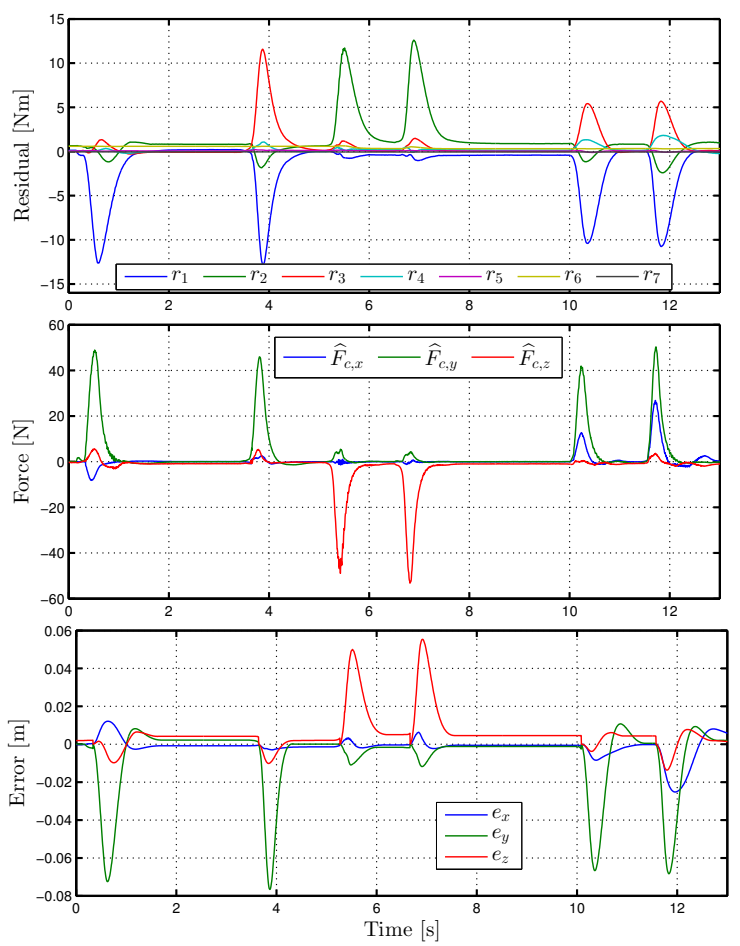

Fig. 5. Contact impedance control with desired inertia matrix at the contact equal to the natural one. Residual vector components [top]. Estimated contact force components [center]. Contact position error components [bottom].

i.e., different from the natural one (which was also coupled). Non-uniform values were chosen on the diagonal, so as to obtain different behaviors along the three Cartesian axes see also Fig. 4. To enhance this effect, the human pushes the robot always on link 6 , although in different directions. The other impedance parameters used were the same as in Sec. VA. In this case, the estimated contact forces $\widehat{\boldsymbol{F}}_{c}$ are needed by the control law in order to obtain the desired mass-springdamper system. Figure 6 shows the same quantities of the previous impedance control experiment. When the contact is initially detected, the robot will move the contact point with a dynamics that depends on the direction of the external force. When the hand is removed, the robot brings back the contact point to the original initial position. As shown in Fig. 6 (third 
plot), the error returns closer to zero on the $y$ axis, where $M_{d, y}=3$. By increasing the desired inertia, the error natural frequency and damping decrease, as indicated by (13), and transients will worsen (see, e.g., the $x$ axis at $t=15 \mathrm{~s}$ ).

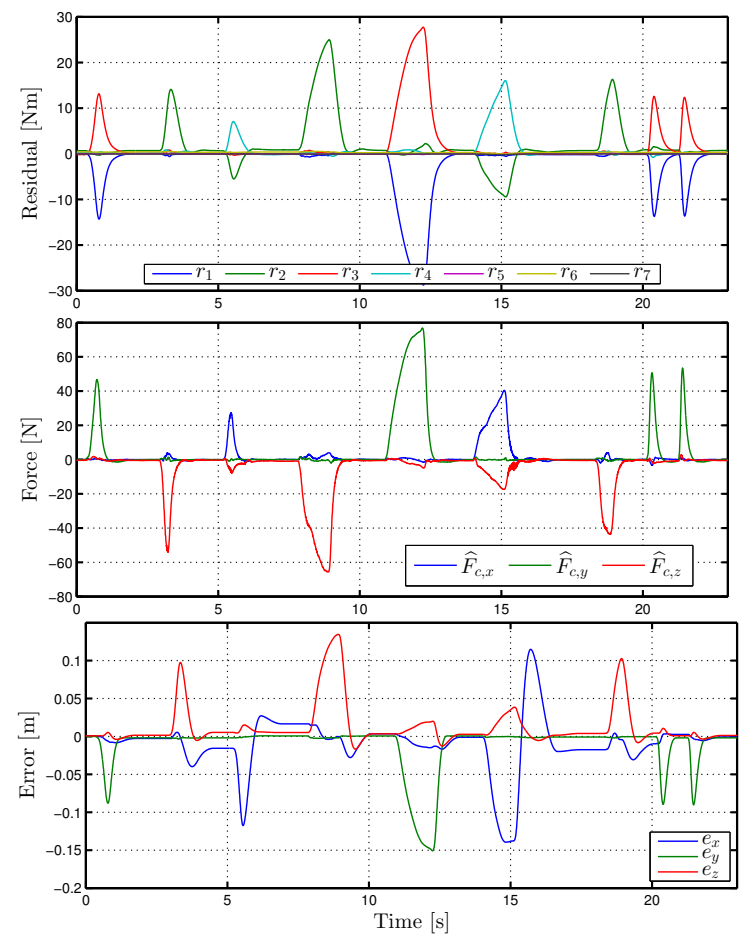

Fig. 6. Contact impedance control with modified inertia matrix at the contact. Residual vector components [top]. Estimated contact force components [center]. Contact position error components [bottom].

\section{Contact force control with possible drift effects}

The purpose of this experiment was to show that it is highly not recommended to try to regulate human-robot contact forces at arbitrary values and in multiple fixed directions of the Cartesian space. When the human does not push or resist along a direction where a non-zero force reference has been specified, the robot typically drifts in space in the attempt to regulate the incompatible desired force. This could be dangerous because an unexpected movement occur, through which the robot may collide with the human. With this in mind, the parameters were set to $\boldsymbol{K}_{d}=10.5 \cdot \boldsymbol{I}_{3}$, $\boldsymbol{K}_{f}=4.1 \cdot \boldsymbol{I}_{3}, \boldsymbol{K}_{N}=15 \cdot \boldsymbol{I}_{7}$, and the desired contact force was chosen as $\boldsymbol{F}_{d}=\left(\begin{array}{lll}0 & 15 & 0\end{array}\right)^{T}$, i..e., only in the $Y$ direction. Figure 8 shows the behavior of the residual vector $\boldsymbol{r}$, of the contact force estimate $\widehat{F}_{c}$, and of the Cartesian velocity $\dot{\boldsymbol{x}}_{c}$ of the contact point. When the human pushes the robot (on link 6) along the $Y$ axis, the robot reacts properly and regulate the force components to the desired level. However, when the human pushes the robot along the $X$ direction, a drift occurs along the $Y$ direction, as shown in Fig. 8 (e.g., at $t=14.8 \mathrm{~s}$ ).

\section{Contact force control with task compatibility}

The contact force control scheme (29) was used in this interaction control experiment, where the human pushes the

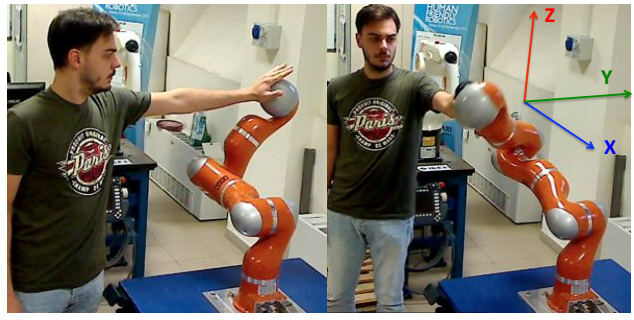

Fig. 7. The set-up and the Cartesian reference frame for the contact force control experiments. On the left, the human is pushing along the $Y$ direction where a non-zero desired force is specified $\left(F_{y, d}=15 \mathrm{~N}\right)$. On the right, a drift effect occurs due the absence of a human reaction in the $Y$ direction (a force is applied approximately along the $X$ direction only).
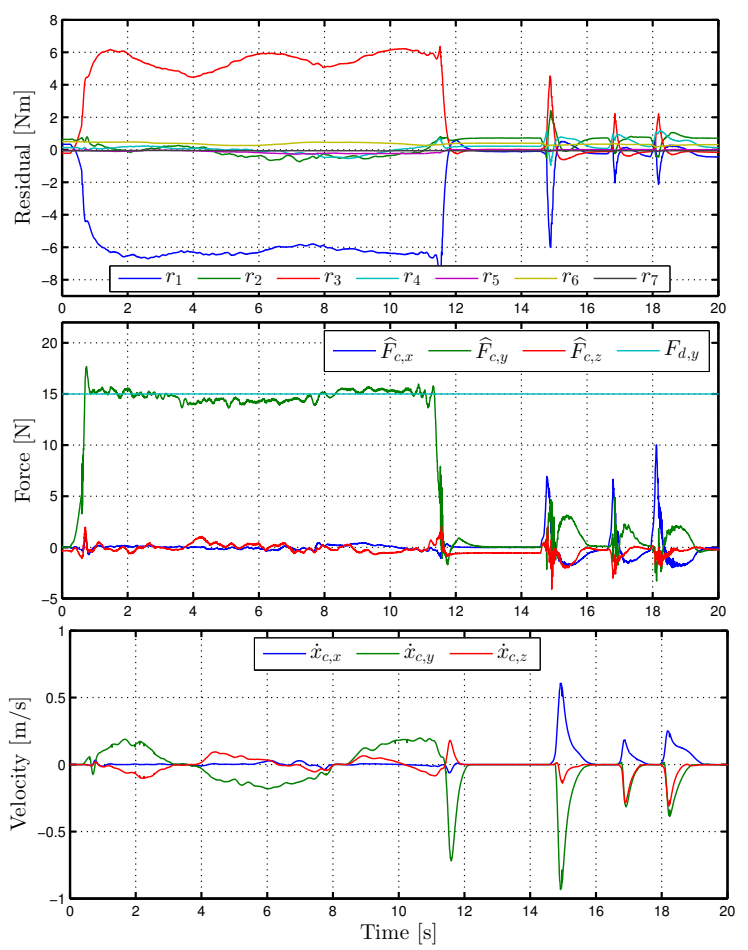

Fig. 8. Contact force control with drift motion due to task incompatibility. Components of the residual vector [top]. Modulus of the estimated contact force [center]. Components of the Cartesian contact velocity $\dot{\boldsymbol{x}}_{c}$ [bottom]. When the human pushes along the $Y$ direction, regulation occurs (see the first $12 \mathrm{~s}$ ). The human pushing along the $X$ direction (where $F_{x, d}=0$ ) will be accommodated by a robot retraction, whereas the absence of a human reaction in the $Y$ direction (where $F_{y, d}=15 \mathrm{~N}$ ) does not allow force regulation and generates large velocity drifts in that same direction (see the last $6 \mathrm{~s}$ in the third plot).

robot successively on link 3, link 5 , and then link 6 (see the associated behaviors of the residuals in Fig. 9, [top]). To avoid incompatibility with the human, only the norm of the desired contact force $\boldsymbol{F}_{d}$ has been, here as a constant $\left\|\boldsymbol{F}_{d}\right\|=15 \mathrm{~N}$. However, $\boldsymbol{F}_{d}$ will change direction according to the human-robot contact type, and being always aligned with the estimated contact force vector which may now vary without any restriction. We will have thus,

$$
F_{d, x}=15 \frac{\widehat{F}_{c, x}}{\left\|\widehat{\boldsymbol{F}}_{c}\right\|}, \quad F_{d, y}=15 \frac{\widehat{F}_{c, y}}{\left\|\widehat{\boldsymbol{F}}_{c}\right\|}, \quad F_{d, z}=15 \frac{\widehat{F}_{c, z}}{\left\|\widehat{\boldsymbol{F}}_{c}\right\|} .
$$


The other force control parameters used were the same as in Sec. V-C. Figure 9 shows the same quantities of the previous force control experiment. Note that the controller starts operating after the first detection of a contact. When the human pushes the robot, it gets compliant in order to regulate the contact force at the desired value. When the human tries to recede, the robot pushes against the human hand so as to maintain the desired $\widehat{\boldsymbol{F}}_{c}=\boldsymbol{F}_{d}$. The fact that the human may abandon the contact and then resume it in a different point and along a different direction is no longer a problem for this correctly defined contact force controller. On the other hand, this force control law is able to regulate contact forces only in static conditions (i.e., when $\dot{\boldsymbol{x}}_{c}=0$ ). While the human is moving the robot during a contact, some error will result as shown in Fig. 9.
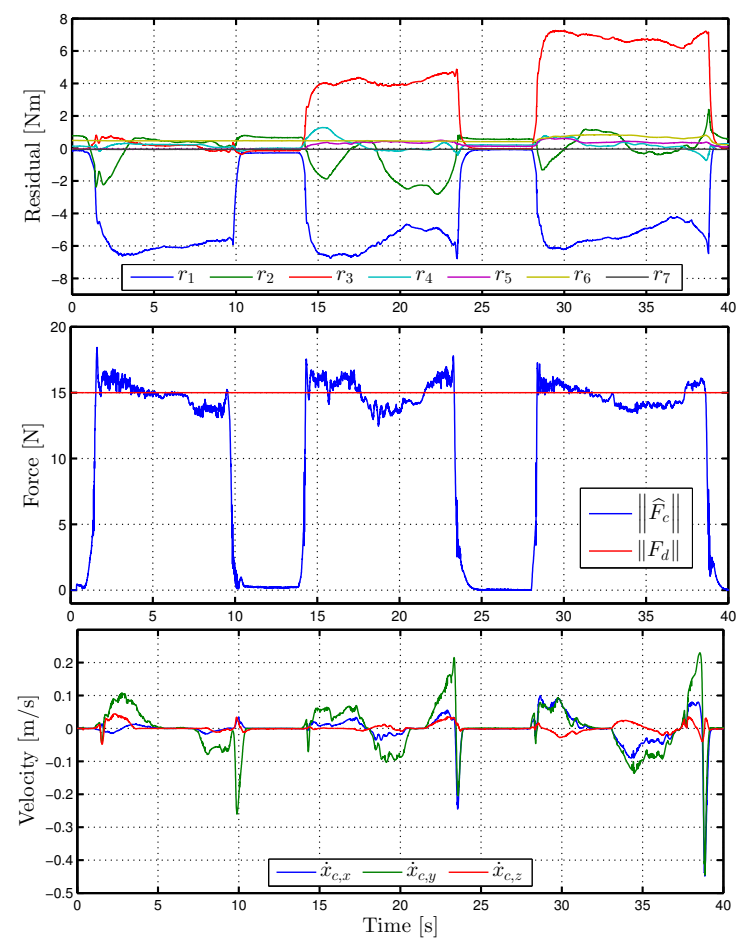

Fig. 9. Contact force control is here designed for automatic task compatibility. Components of the residual vector [top]. Modulus of the estimated contact force [center]. Components of the Cartesian contact velocity $\dot{\boldsymbol{x}}_{c}$ [bottom].

\section{CONCLUSION}

We have expanded the portfolio of controllers for physical human-robot interaction tasks, introducing the concept of motion and/or force control at any contact point on the robot. Generalizations of classical impedance and direct force control schemes have been introduced and implemented without the explicit need of a force sensor, relying on a fast estimation of contact forces. Beside developing and refining further such basic robotic skills for safe collaboration, our next step will be their application to concrete and specific tasks (e.g, performing human-robot collaborative assembly) within the industrial use cases of the SAPHARI project. We also wish to exploit the robot redundancy for controlling motion in the plane perpendicular to the estimated contact force (i.e., achieving hybrid force-motion control).

\section{REFERENCES}

[1] "KUKA presents the world's first lightweight industrial robot at Hanover Fair," April 10, 2013. [Online]. Available: http://www.kukalabs.com/en/pressevents/news/201304_LBR_iiwa_world_premiere.htm

[2] "ABB unveils collaborative robot: YuMi," September 9, 2014 [Online]. Available: http://www.roboticstoday.com/news/abb-unveilscollaborative-robot-yumi-3041

[3] O. Khatib, K. Yokoi, O. Brock, K.-S. Chang, and A. Casal, "Robots in human environments: Basic autonomous capabilities," Int. J. of Robotics Research, vol. 18, no. 7, pp. 684-696, 1999.

[4] J. Heinzmann and A. Zelinsky, "Quantitative safety guarantees for physical human-robot interaction," Int. J. of Robotics Research, vol. 22, no. 7/8, pp. 479-504, 2003.

[5] A. Bicchi and G. Tonietti, "Fast and soft arm tactics: Dealing with the safety-performance trade-off in robot arms design and control," IEEE Robotics and Automation Mag., vol. 11, no. 2, pp. 22-33, 2004.

[6] A. De Santis, B. Siciliano, A. De Luca, and A. Bicchi, "An atlas of physical human-robot interaction," Mechanism and Machine Theory, vol. 43, no. 3, pp. 253-270, 2008.

[7] A. De Luca and R. Mattone, "Sensorless robot collision detection and hybrid force/motion control," in Proc. IEEE Int. Conf. on Robotics and Automation, 2005, pp. 1011-1016.

[8] A. De Luca, A. Albu-Schäffer, S. Haddadin, and G. Hirzinger, "Collision detection and safe reaction with the DLR-III lightweight robot arm," in Proc. IEEE/RSJ Int. Conf. on Intelligent Robots and Systems, 2006, pp. 1623-1630.

[9] A. De Luca and F. Flacco, "Integrated control for pHRI: Collision avoidance, detection, reaction and collaboration," in Proc. IEEE Int. Conf. on Biomed. Robotics and Biomechatronics, 2012, pp. 288-295.

[10] S. Haddadin, A. Albu-Schäffer, A. De Luca, and G. Hirzinger, "Collision detection and reaction: A contribution to safe physical humanrobot interaction," in Proc. IEEE/RSJ Int. Conf. on Intelligent Robots and Systems, 2008, pp. 3356-3363.

[11] M. Erden and T. Tomiyama, "Human-intent detection and physically interactive control of a robot without force sensors," IEEE Trans. on Robotics, vol. 26, no. 2, pp. 370-382, 2010 .

[12] M. Geravand, F. Flacco, and A. De Luca, "Human-robot physical interaction and collaboration using an industrial robot with a closed control architecture," in Proc. IEEE Int. Conf. on Robotics and Automation, 2013, pp. 4000-4007.

[13] D. Ebert and D. Henrich, "Safe human-robot-cooperation: Imagebased collision detection for industrial robots," in Proc. IEEE/RSJ Int. Conf. on Intelligent Robots and Systems, 2002, pp. 239-244.

[14] F. Flacco, T. Kröger, A. De Luca, and O. Khatib, "A depth space approach to human-robot collision avoidance," in Proc. IEEE Int. Conf. on Robotics and Automation, 2012, pp. 338-345.

[15] E. Magrini, F. Flacco, and A. De Luca, "Estimation of contact forces using a virtual force sensor," in Proc. IEEE/RSJ Int. Conf. on Intelligent Robots and Systems, 2014, pp. 2126-2133.

[16] N. Hogan, "Impedance control: An approach to manipulation: Part I - Theory, Part II - Implementation, Part III - Applications," ASME J. of Dynamic Systems, Measurement, and Control, vol. 107, pp. 1-24, 1985.

[17] B. Siciliano and L. Villani, Robot Force Control. Kluwer, 1999.

[18] O. Khatib, "A unified approach for motion and force control of robot manipulators: The operational space formulation," IEEE Trans. on Robotics and Automation, vol. 3, no. 1, pp. 43-53, 1987.

[19] B. Siciliano, L. Sciavicco, L. Villani, and G. Oriolo, Robotics: Modeling, Planning and Control, 3rd ed. London: Springer, 2008.

[20] A. De Luca and W. Book, "Robots with flexible elements," in Springer Handbook of Robotics, B. Siciliano and O. Khatib, Eds. Springer, 2008, pp. 287-319.

[21] KUKA.FastResearchInterface 1.0, KUKA System Technology (KST), Augsburg, D, 2011, Version 2.

[22] A. Albu-Schäffer, C. Ott, and G. Hirzinger, "A unified passivitybased control framework for position, torque and impedance control of flexible joint robots," Int. J. of Robotics Research, vol. 26, pp. 23-39, 2007.

[23] C. Gaz, F. Flacco, and A. De Luca, "Identifying the dynamic model used by the KUKA LWR: A reverse engineering approach," in Proc. IEEE Int. Conf. on Robotics and Automation, 2014, pp. 1386-1392. 